cohort, and tended to favor enoxaparin in the streptokinase cohort.

In the second paper, Sabatine et al. compared the efficacy and safety profiles of enoxaparin and UFH in patients treated with and without clopidogrel in order to determine whether enoxaparin and clopidogrel can be used together. The authors evaluated 2,173 patients who received clopidogrel (at the treating physician's discretion) and 12,918 who did not; patients who underwent percutaneous coronary intervention during the index hospitalization were excluded from the analysis. Compared with UFH, enoxaparin reduced the 30-day rate of a composite end point (death, recurrent $\mathrm{MI}$, recurrent myocardial ischemia, or stroke) both in patients who received clopidogrel (10.8\% vs $13.9 \%$; OR $0.70,95 \% \mathrm{Cl} 0.53-0.93$ ) and in those who did not (13.3\% vs 15.3\%; OR 0.85, 95\% Cl 0.76-0.94). The 30-day rate of major bleeding was higher in the enoxaparin group than in the UFH group in both the clopidogrel and the non-clopidogrel cohorts. This rate was, however, not significantly higher in patients who received clopidogrel than in those who did not $(P=0.61$ for the interaction). Clopidogrel use was not associated with increased incidence of intracranial hemorrhage. Net clinical benefit favored use of enoxaparin over UFH, with or without concomitant clopidogrel.

These analyses show that enoxaparin is superior to UFH for support of fibrinolysis in STEMI, irrespective of the lytic agent used; clopidogrel can safely be used alongside enoxaparin as part of a reperfusion strategy.

Original articles Giraldez RR et al. (2007) Enoxaparin is superior to unfractionated heparin in patients with ST elevation myocardial infarction undergoing fibrinolysis regardless of the choice of lytic: an ExTRACT-TIMI 25 analysis. Eur Heart J 28: 1566-1573

Sabatine MS et al. (2007) Efficacy and safety of enoxaparin versus unfractionated heparin in patients with ST-segment elevation myocardial infarction also treated with clopidogrel. J Am Coll Cardiol 49: 2256-2263

\section{Use of ICDs for primary prevention following acute MI}

In MADIT II, defibrillator implantation after myocardial infarction (MI) reduced sudden cardiac death by $67 \%$ in patients with a left ventricular ejection fraction (LVEF) of $30 \%$ or less. The FDA consequently approved implantable cardioverter-defibrillator (ICD) use in patients meeting the entry criteria for this trial, but there is still some controversy over the costeffectiveness of ICDs in this patient group, and over the LVEF cutoff used to determine whether a device should be implanted.

Yap and co-workers pooled data from the placebo arms of four studies of patients with LVEF $\leq 40 \%$ after acute MI $(n=2,828$ patients; mean age 65 years). LVEF progressively decreased over 2 years of follow-up. LVEF did not predict short-term ( $<45$-day) mortality, but between 45 days and 2 years each $10 \%$ reduction in LVEF was associated with significant increases in all-cause mortality, arrhythmic cardiac mortality and nonarrhythmic cardiac mortality (by $42 \%, 39 \%$ and $49 \%$, respectively; $P<0.001$ for all). No single dichotomy limit was satisfactory for determining the need for ICD treatment. Patients with an LVEF of $16-20 \%$ were most likely to benefit from ICD treatment-arrhythmic cardiac deaths outweighed nonarrhythmic deaths in this patient group, and the number needed to treat to prevent one arrhythmic death was 4.3. This figure rose, however, as LVEF decreased further; all deaths in patients with LVEF $\leq 10 \%$ were from nonarrhythmic causes, and ICD treatment would, therefore, not be cost-effective in this patient group. In light of these findings, the authors advocate risk assessment for arrhythmic and nonarrythmic mortality on a patient-by-patient basis.

Original article Yap YG et al. (2007) Optimising the dichotomy limit for left ventricular ejection fraction in selecting patients for defibrillator therapy after myocardial infarction. Heart 93: 832-836

\section{OPERA registry provides support for the widened ESC/ACC definition of MI}

In 2000, the Joint European Society of Cardiology/American College of Cardiology (ESC/ACC) Committee on the redefinition of myocardial infarction (MI) widened the definition of $\mathrm{MI}$ to include any amount of necrosis caused by ischemia, thereby encompassing non-ST-segment elevation MI (non-STEMI). Data reported in a recent paper by the OPERA Investigators support this new definition, demonstrating similar outcomes for patients with STEMI and those with non-STEMI. 